\title{
Robotic-assisted hysterectomy: patient selection and perspectives
}

\author{
This article was published in the following Dove Press journal: \\ International Journal of Women's Health \\ 23 March 2017 \\ Number of times this article has been viewed
}

\author{
Noam Smorgick \\ Departments of Obstetrics and \\ Gynecology, Assaf Harofe Medical \\ Center, Affiliated with the Sackler \\ Faculty of Medicine, Tel Aviv \\ University, Tel Aviv, Israel
}

\begin{abstract}
Minimally invasive hysterectomy via the laparoscopic or vaginal approach is beneficial to patients when compared with laparotomy, but has not been offered in the past to all women because of the technical difficulties and the long learning curve required for laparoscopic hysterectomy. Robotic-assisted hysterectomy for benign indications may allow for a shorter learning curve but does not offer clear advantages over conventional laparoscopic hysterectomy in terms of surgical outcomes. In addition, robotic hysterectomy is invariably associated with increased costs. Nevertheless, this surgical approach has been widely adopted by gynecologic surgeons. The aim of this review is to describe specific indications and patients who may benefit from robotic-assisted hysterectomy. These include hysterectomy for benign conditions in cases with high surgical complexity (such as pelvic adhesive disease and endometriosis), hysterectomy and lymphadenectomy for treatment of endometrial carcinoma, and obese patients. In the future, additional evidence regarding the benefits of single-site robotic hysterectomy may further modify the indications for robotic-assisted hysterectomy.
\end{abstract}

Keywords: robotic-assisted hysterectomy, single-site laparoscopy, minimally invasive hysterectomy

\section{Introduction}

The minimally invasive surgical approach to hysterectomy (including vaginal hysterectomy, laparoscopic hysterectomy, and robotic-assisted hysterectomy) has advantages over laparotomic hysterectomy in the rates of short- and long-term complications. ${ }^{1}$ However, the vaginal approach to hysterectomy is not feasible in many women undergoing hysterectomy owing to large uterine size, prior pelvic surgeries, need for concomitant adnexal surgery, and malignancy. Thus, the minimally invasive approach appropriate for these cases is either laparoscopic or robotic hysterectomy. For uncomplicated benign hysterectomy cases, the outcomes and complications of laparoscopic and robotic hysterectomy are comparable, while the cost of robotic hysterectomy is higher. ${ }^{2}$ Thus, in this patient group, there is no clear advantage of robotic over laparoscopic hysterectomy. However, some patients with complex surgical pathology (such as advanced endometriosis and extensive pelvic adhesions), obesity, and those with endometrial carcinoma may benefit from robotic-assisted hysterectomy. In addition, surgical advancements such as single-site hysterectomy may change the relative advantages and disadvantages of laparoscopic versus robotic hysterectomy. In this review, we will describe the evidence and rationale for the selection of patients who are likely to benefit from robotic hysterectomy.

\footnotetext{
Correspondence: Noam Smorgick Gynecologic Endoscopy Unit, Departments of Obstetrics and Gynecology, Assaf Harofe Medical Center, Zerifin, 70300, Israel Tel +972 89779000 Fax +97289778026 Email noam_yossi@yahoo.com
} 


\section{Robotic versus laparoscopic approach for simple hysterectomy}

To date, two published randomized controlled trials compared laparoscopic and robotic hysterectomy in uncomplicated benign cases, ${ }^{3,4}$ and one randomized controlled trial compared either laparoscopic or vaginal hysterectomy with robotic hysterectomy. ${ }^{5}$ Overall, no differences were found between groups in complications rates, estimated blood loss, and hospital stay. Similarly, conversion rates to laparotomy were similar between groups. However, two of the studies reported longer operative time for the robotic hysterectomy group compared with the laparoscopic group. ${ }^{2,3}$ These trials were criticized for a bias in favor of the nonrobotic methods, ie, the surgeons were more experienced in laparoscopic and vaginal surgery than with robotic hysterectomies. Based on these studies, a recent Cochrane review ${ }^{2}$ and a recent meta-analysis ${ }^{6}$ concluded that the current evidence does not demonstrate any clinically meaningful differences in surgical outcomes between robotic and laparoscopic hysterectomy for benign disease. Nevertheless, the use of the robotic platform and the number of hysterectomies performed robotically in the US continues to increase, reaching 263,000 cases in $2015 .^{7}$ At the same time, the increased rates of robotic hysterectomy have probably contributed to the decreased rates of laparotomic and vaginal hysterectomy. ${ }^{8}$ This trend likely points to the individual preference of many gynecologic surgeons for the use of the robotic platform over the standard laparoscopic approach. Possible explanations for this preference could be the technical advantages of the robotic platform, including the 3-dimensional vision, wristed instrumentation, tremor reduction, and comfortable ergonomics, in addition to the drawbacks of the conventional laparoscopy, namely, the long learning curve and relatively advanced laparoscopic skills required for laparoscopic hysterectomy.

\section{Robotic versus laparoscopic approach for "complex" hysterectomy}

The definition of "complex" hysterectomy is strongly influenced by surgeons' surgical skills and is likely to differ between different surgeons. However, most surgeons will agree that cases with extensive pelvic adhesive disease, advanced stage endometriosis, and uterine fibroids located in the lower uterine segments, or significantly increasing uterine size would present more challenging hysterectomies. In the past, those patients have not been considered candidates for minimally invasive hysterectomy. With the advent of the robotic platform, on the one hand, and the advances in laparoscopic skills and instrumentation, on the other, minimally invasive hysterectomy has become a viable option for these patients. Boggess et $\mathrm{al}^{9}$ reported on the outcomes of 152 women who underwent robotic hysterectomy for "complex" benign pathology, including prior abdominal/ pelvic surgeries, endometriosis, and uterine fibroids. The rates of intraoperative and postoperative complications were low, and no conversions to laparotomy were required. Similarly, Lim et $\mathrm{al}^{10}$ compared the outcomes of 2,300 robotic, 9,745 abdominal, 8,121 vaginal, and 11,952 laparoscopic hysterectomies performed by high-volume surgeons. Although women in the robotic cohort were more likely to have a "complex" pathology (including higher rates of pelvic adhesions, obesity, and large uteri $[>250 \mathrm{~g}]$ ), fewer intraoperative complications were noted when compared with the abdominal and vaginal cohorts, and fewer postoperative complications when compared with all the other cohorts.

Hysterectomy in cases of advanced stage endometriosis probably represents the most difficult form of simple extrafascial hysterectomy. These surgeries typically involve extensive adhesiolysis, dissection of the rectovaginal septum, and ureterolysis and often include resection of deep infiltrating endometriosis in addition to the hysterectomy. Although no study to date specifically reports the outcomes of robotic hysterectomy in cases of advanced endometriosis, some studies reporting on the overall outcomes of robotic hysterectomy include cases with advanced stage endometriosis. Patzkowsky et $\mathrm{al}^{11}$ retrospectively compared outcomes of 288 robotic hysterectomies with 257 laparoscopic hysterectomies. In the robotic cohort, $15.3 \%$ had stage III-IV endometriosis, versus $4.7 \%$ in the laparoscopic cohort. Patients with endometriosis were more likely to develop vaginal cuff abscess compared with patients without endometriosis. Furthermore, a correlation was noted between the severity of endometriosis and the risk of vaginal cuff abscess, reaching $9.3 \%$ in patients with stage III-IV endometriosis. Magrina et al ${ }^{12}$ describe 493 cases of advanced stage endometriosis undergoing surgical excision via the laparoscopic $(\mathrm{n}=162)$ or robotic-assisted $(\mathrm{n}=331)$ approach. In the robotic group, almost $50 \%$ of the patients underwent hysterectomy in addition to endometriosis resection, compared with $27.1 \%$ in the laparoscopic group. Among the hysterectomy cases from both cohorts, intra- and postoperative complications were low, as was the rate of conversion. Overall, surgical outcomes of the laparoscopic and robotic cohorts were comparable, although robotic surgeries required longer operating time, possibly because of the higher rate of hysterectomy in this cohort. Other retrospective studies investigating the outcomes of fertility sparing surgery 
for advanced stage endometriosis using the robotic or laparoscopic approach did not find significant differences between these approaches. ${ }^{13,14}$ However, most of these studies were conducted in tertiary care centers, and the operating surgeons were highly skilled in conventional laparoscopy. Thus, there is no definite benefit of the robotic platform over conventional laparoscopy for performing "complex" hysterectomies, but individual surgeons may find its advantages useful for completing these challenging cases.

\section{Robotic versus laparoscopic approach for treatment of endometrial carcinoma}

The introduction of the robotic platform in gynecologic oncology allowed many patients the option of a minimally invasive procedure, which was available to relatively few patients in the past. Although hysterectomy and pelvic/paraaortic lymphadenectomy for treatment of endometrial cancer is possible via conventional laparoscopy, and provides shorter hospital stay and fewer complications when compared with laparotomy, ${ }^{15}$ in practice this approach has not been offered to the majority of patients with endometrial cancer. The reasons for the low adoption of the conventional laparoscopic approach for treatment of endometrial carcinoma included the long learning curve and advanced laparoscopic skills required for performing laparoscopic lymphadenectomy as well as the high rate of obesity among patients. The robotic platform may help in overcoming these challenges.

Several studies have investigated the learning curve for laparoscopic and robotic lymphadenectomy. Rocconi et al, ${ }^{16}$ using the cumulative sum control chart analysis to study the learning curve for robotic hysterectomy and robotic pelvic lymphadenectomy, demonstrated a learning curve of 14 cases and 19 cases, respectively. Lim et $\mathrm{al}^{17}$ compared the learning curve for hysterectomy and pelvic lymphadenectomy via the robotic and laparoscopic approaches by measuring operative time. They found that the learning curve for the robotic procedure was shorter than for the laparoscopic procedure: the proficiency for the robotic procedure was reached after 24 cases, while that for the laparoscopic procedure was reached after 49 cases. Furthermore, the learning curve for the robotic procedure plateaued after the 24th case, while the learning curve for the laparoscopic procedure did not plateau even after 49 cases. Para-aortic lymphadenectomy, which is sometimes required in patients with endometrial carcinoma, may also be completed robotically, using the fourth robotic arm and placing the robotic ports relatively cephalad in the torso. ${ }^{18}$
A specific subgroup of patients with endometrial carcinoma who may benefit significantly from the robotic approach is the patients with obesity, ie, body mass index (BMI) above $35 \mathrm{~kg} / \mathrm{m}^{2}$. These patients, who are at risk for endometrial carcinoma, have the highest rates of morbidity when undergoing laparotomy. ${ }^{19,20}$ Conventional laparoscopic hysterectomy is feasible in these cases, but rate of conversion to laparotomy is high, reaching $57 \%$ in patients with BMI $>40 \mathrm{~kg} / \mathrm{m}^{2}$, possibly owing to the restriction in laparoscopic movements in obese patients. ${ }^{15}$ Although no randomized controlled study is available to compare the conversion rate of laparoscopic and robotic hysterectomy in obese patients with endometrial cancer, retrospective series (sometimes using historical laparoscopic cohorts) showed low conversion rate with the robotic route, in the range of $5 \% .{ }^{21}$ As a result, the robotic route has become the main minimally invasive surgical approach in obese patients with endometrial carcinoma. Chan et al, ${ }^{22}$ using the Nationwide Inpatient Sample data from 2011, reported that $48 \%$ of obese endometrial carcinoma patients underwent a minimally invasive surgical procedure, and in $81 \%$ of these cases, the robotic route was chosen.

\section{Robotic versus laparoscopic approach for hysterectomy in obese patients}

As described earlier for obese patients with endometrial carcinoma undergoing a robotic procedure, obese patients with benign conditions requiring hysterectomy may also benefit from the robotic approach. A meta-analysis by Iavazzo and Gkegkes ${ }^{23}$ described the outcomes of robotic hysterectomy for various indications (benign and malignant) in obese patients with BMI $>30 \mathrm{~kg} / \mathrm{m}^{2}$. Overall complications were low, and included conversion to laparotomy in $4.1 \%$, wound complications in $2.4 \%$, vaginal cuff complications in $0.9 \%$, and need for blood transfusions in $0.9 \%$.

\section{Single-site hysterectomy via laparoscopic and robotic approach}

Single-site laparoscopic surgery has been introduced as an alternative to multiport laparoscopic surgery, with the aim of reducing port number and consequently postoperative pain, as well as improved cosmesis. This surgical approach has not yet gained widespread acceptance among minimally invasive surgeons owing to the technical difficulty in maneuvering the laparoscopic instruments through the single port, as a result of the limited triangulation. The robotic version of single-site laparoscopic surgery is available for the da 
Vinci platform, using specially adapted robotic ports and instruments. The single-site robotic platform is not identical to the multiport robotic platform since the instruments are not fully wristed and the degrees of movements are more limited, but it is more comfortable to use when compared to the single-site laparoscopic instruments because it does allow for some triangulation. Thus, laparoscopic suturing with the single-site robotic platform is somewhat technically challenging, and some surgeons opt to suture the vaginal cuff by transvaginal closure. ${ }^{24}$

The current literature, which at this time includes retrospective case series, describes the outcomes of single-site robotic hysterectomy in comparison with multiport robotic hysterectomy. Several studies describe the feasibility of single-site robotic hysterectomy for benign conditions, and single-site hysterectomy and lymphadenectomy for endometrial carcinoma. ${ }^{24,25}$ Bogliolo et $\mathrm{al}^{24}$ compared single-site and multiport robotic hysterectomy for benign conditions. The main advantage of the single-site robotic hysterectomy in their study was reduced cost, with some additional benefit in the short-term postoperative pain. The cost saving was attributed to the use of two robotic instruments per procedure in the single-site surgery as opposed to three robotic instruments in the multiport procedure, and amounted to $\$ 1,500-\$ 2,000$ per case. Another advantage of the robotic single-site platform may be its shorter learning curve when compared with single-site laparoscopic hysterectomy. ${ }^{26}$ At this time, the preliminary outcomes reported for single-site robotic hysterectomy appear promising, but most authors agree that more studies are needed to define the appropriate applications for this approach prior to widespread adoption. ${ }^{27}$ In particular, at this time, the single-site robotic platform is unlikely to replace the multiport robotic platform when operating cases with high surgical complexity.

\section{Conclusion}

Robotic-assisted hysterectomy has gained increased popularity over the last decade. The adoption of this surgical approach has enabled many patients to undergo minimally invasive hysterectomy. However, there is no clear evidence that robotic hysterectomy is superior to conventional laparoscopic hysterectomy in patients with benign conditions and moderate surgical complexity, while the cost of the robotic procedure remains higher. ${ }^{28}$ Nevertheless, some patients may benefit from robotic procedures, including patients with endometrial carcinoma, obesity, and patients with benign conditions involving high surgical complexity such as advanced stage endometriosis and pelvic adhesive disease. The more recent availability of the single-site robotic platform may prove to be another benefit of this surgical approach.

\section{Disclosure}

The author reports no conflicts of interest in this work.

\section{References}

1. Aarts JW, Nieboer TE, Johnson N, et al. Surgical approach to hysterectomy for benign gynaecological disease. Cochrane Database Syst Rev. 2015;8:CD003677.

2. Liu H, Lawrie TA, Lu D, Song H, Wang L, Shi G. Robot-assisted surgery in gynaecology. Cochrane Database Syst Rev. 2014;12: CD011422.

3. Paraiso MF, Ridgeway B, Park AJ, et al. A randomized trial comparing conventional and robotically assisted total laparoscopic hysterectomy. Am J Obstet Gynecol. 2013;208:368.e1-e7.

4. Sarlos D, Kots L, Stevanovic N, von Felten S, Schär G. Robotic compared with conventional laparoscopic hysterectomy: a randomized controlled trial. Obstet Gynecol. 2012;120:604-611.

5. Lönnerfors C, Reynisson P, Persson J. A randomized trial comparing vaginal and laparoscopic hysterectomy vs robot-assisted hysterectomy. J Minim Invasive Gynecol. 2015;22:78-86.

6. Albright BB, Witte T, Tofte AN, et al. Robotic versus laparoscopic hysterectomy for benign disease: a systematic review and meta-analysis of randomized trials. J Minim Invasive Gynecol. 2016;23:18-27.

7. Simpson KM, Advincula AP. The essential elements of a roboticassisted laparoscopic hysterectomy. Obstet Gynecol Clin North Am. 2016;43:479-493.

8. Wright JD, Herzog TJ, Tsui J, et al. Nationwide trends in the performance of inpatient hysterectomy in the United States. Obstet Gynecol. 2013;122:233-241.

9. Boggess JF, Gehrig PA, Cantrell L, et al. Perioperative outcomes of robotically assisted hysterectomy for benign cases with complex pathology. Obstet Gynecol. 2009;114:585-593.

10. Lim PC, Crane JT, English EJ, et al. Multicenter analysis comparing robotic, open, laparoscopic, and vaginal hysterectomies performed by high-volume surgeons for benign indications. Int J Gynaecol Obstet. 2016;133:359-364.

11. Patzkowsky K, As-Sanie S, Smorgick N, Song AH, Advincula AP. Perioperative outcomes of robotic versus laparoscopic hysterectomy for benign disease. JSLS. 2013;17:100-106.

12. Magrina JF, Espada M, Kho RM, Cetta R, Chang YH, Magtibay PM. Surgical excision of advanced endometriosis: perioperative outcomes and impacting factors. J Minim Invasive Gynecol. 2015;22:944-950.

13. Nezhat CR, Stevens A, Balassiano E, Soliemannjad R. Robotic-assisted laparoscopy vs conventional laparoscopy for the treatment of advanced stage endometriosis. J Minim Invasive Gynecol. 2015;22:40-44.

14. Chen SH, Li ZA, Du XP. Robot-assisted versus conventional laparoscopic surgery in the treatment of advanced stage endometriosis: a metaanalysis. Clin Exp Obstet Gynecol. 2016;43:422-426.

15. Walker JL, Piedmonte MR, Spirtos NM, et al. Laparoscopy compared with laparotomy for comprehensive surgical staging of uterine cancer: Gynecologic Oncology Group Study LAP2. J Clin Oncol. 2009;27: 5331-5336.

16. Rocconi RP, Meredith C, Finan MA. Evaluation of the learning curve of total robotic hysterectomy with or without lymphadenectomy for a gynecologic oncology service. J Robot Surg. 2011;5:189-193.

17. Lim PC, Kang E, Park DH. Learning curve and surgical outcome for robotic-assisted hysterectomy with lymphadenectomy: case-matched controlled comparison with laparoscopy and laparotomy for treatment of endometrial cancer. J Minim Invasive Gynecol. 2010;17: 739-748.

18. Seamon LG, Cohn DE, Richardson DL, et al. Robotic pelvic and aortic lymphadenectomy for endometrial cancer: the console surgeon's perspectives on surgical technique and directing the assistant. J Minim Invasive Gynecol. 2010;17:180-185. 
19. Heo M, Kabat GC, Strickler HD, et al. Optimal cutoffs of obesity measures in relation to cancer risk in postmenopausal women in the Women's Health Initiative Study. J Womens Health. 2015;24: 218-227.

20. Hinshaw SJ, Gunderson S, Eastwood D, Bradley WH. Endometrial carcinoma: the perioperative and long-term outcomes of robotic surgery in the morbidly obese. J Surg Oncol. 2016;114:884-887.

21. Gaia G, Holloway RW, Santoro L, Ahmad S, Di Silverio E, Spinillo A. Robotic-assisted hysterectomy for endometrial cancer compared with traditional laparoscopic and laparotomy approaches: a systematic review. Obstet Gynecol. 2010;116:1422-1431.

22. Chan JK, Gardner AB, Taylor K, et al. Robotic versus laparoscopic versus open surgery in morbidly obese endometrial cancer patients - a comparative analysis of total charges and complication rates. Gynecol Oncol. 2015;139:300-305.

23. Iavazzo C, Gkegkes ID. Robotic assisted hysterectomy in obese patients: a systematic review. Arch Gynecol Obstet. 2016;293:1169-1183.
24. Bogliolo S, Ferrero S, Cassani C, et al. Single-site versus multiport robotic hysterectomy in benign gynecologic diseases: a retrospective evaluation of surgical outcomes and cost analysis. J Minim Invasive Gynecol. 2016;23:603-609.

25. Corrado G, Mereu L, Bogliolo S, et al. Robotic single site staging in endometrial cancer: a multi-institution study. Eur J Surg Oncol. 2016; 42:1506-1511

26. Cela V, Freschi L, Simi G, Ruggiero M, Tana R, Pluchino N. Robotic single-site hysterectomy: feasibility, learning curve and surgical outcome. Surg Endosc. 2013;27:2638-2643.

27. Truong M, Kim JH, Scheib S, Patzkowsky K. Advantages of robotics in benign gynecologic surgery. Curr Opin Obstet Gynecol. 2016;28: 304-310.

28. Swenson CW, Kamdar NS, Harris JA, Uppal S, Campbell DA Jr, Morgan DM. Comparison of robotic and other minimally invasive routes of hysterectomy for benign indications. Am J Obstet Gynecol. 2016;215:650.e1-650.e8.
International Journal of Women's Health

\section{Publish your work in this journal}

The International Journal of Women's Health is an international, peerreviewed open-access journal publishing original research, reports, editorials, reviews and commentaries on all aspects of women's healthcare including gynecology, obstetrics, and breast cancer. The manuscript management system is completely online and includes

\section{Dovepress}

a very quick and fair peer-review system, which is all easy to use. Visit http://www.dovepress.com/testimonials.php to read real quotes from published authors.

\footnotetext{
Submit your manuscript here: http://www.dovepress.com/international-journal-of-womens-health-journal
} 